\title{
Quality of life on hemodialysis and inflammation: a descriptive analysis
}

\author{
M.R. Bacci ${ }^{1}$, F. Adami ${ }^{3}$, F.W.S. Figueiredo ${ }^{3}$, B.C.A. Alves ${ }^{2}$, G.L. da Veiga ${ }^{2}$ and F.L.A. Fonseca ${ }^{1,2,4}$ \\ ${ }^{1}$ Departamento de Clínica Médica, Faculdade de Medicina ABC, Santo André, SP, Brasil \\ ${ }^{2}$ Laboratório de Análises Clínicas, Faculdade de Medicina ABC, Santo André, SP, Brasil \\ ${ }^{3}$ Laboratório de Epidemiologia e Análises de Dados, Faculdade de Medicina ABC, Santo André, SP, Brasil \\ ${ }^{4}$ Departamento de Ciências Farmacêuticas, Universidade Federal de São Paulo, Diadema, SP, Brasil
}

\begin{abstract}
Chronic kidney disease (CKD) is highly prevalent worldwide. Patients with CKD on hemodialysis are more likely to present behavioral changes and worse quality of life as a result of their routine and complications. They also have higher levels of cytokines. The aim of this study is to assess the relationship between the inflammatory profile and quality of life measured by KDOQL-SF36 in hemodialysis outpatients. Patients older than 21 years of age and on routine hemodialysis for at least 6 months with treatment on a regular weekly basis were included and their anthropometric parameters and serum inflammatory markers were evaluated. Thirty patients consented to participate. Homocysteine (Hcy) levels were correlated with worse glomerular filtration rate (GFR; $P=0.003$ ) and creatinine $(P=0.002)$. IL-6 was not correlated with worse nutritional status taking into account body mass index $\left(\mathrm{BMl} ; \mathrm{kg} / \mathrm{m}^{2} ; \mathrm{P}=0.83\right)$. On the other hand, TNF-alpha was positively correlated with albumin $(P=0.008)$, nutritional status by $B M I(P=0.04)$, and nutritional status by arm circumference area $(P=0.04)$. IL-6 was correlated with activity limitation $(P=0.02)$ and Hcy with work status $(P=0.04)$. Hcy was correlated with nutritional status and inflammatory markers. In this population, the majority of the sections in KDOQL-SF36 were not correlated with cytokines levels.
\end{abstract}

Key words: Quality of life; Hemodialysis; Inflammation; Homocysteine

\section{Introduction}

The terminal stage of chronic kidney disease (CKD) is characterized by a change in the routine of the individual when dialysis treatment begins. Quality of life assessments through the application of validated questionnaires is a simple and important tool to measure the quality of treatment and the individual's acceptance of the disease $(1,2)$.

Kidney Disease and Quality-of-Life Short-Form 36 (KDQOL-SF36) is a quality-of-life assessment tool for patients with CKD undergoing hemodialysis and peritoneal dialysis, able to subjectively assess some parameters of the patient's daily life that can reflect on their well-being and quality of life. The questionnaire is self-applicable and has a multiitem scale that evaluates health concepts, ranging from limitations on physical activities and health problems, social limitations due to physical and emotional problems, limitations in day-to-day activities due to health problems, pain, and mental health among others (3).

Dialysis patients may present a progressive reduction of their cognitive and intellectual level, nutritional alterations, a higher propensity to infections, and worsening of quality of life, although an improvement of clinical and laboratory conditions has been described (4). One of the hypotheses for changes in cognitive function in CKD patients is the increase in the synthesis of pro-inflammatory mediators $(1,4,5)$. Higher levels of proinflammatory cytokines and other mediators of inflammation have been considered potential agents that favor the reduction of quality of life in patients with CKD on dialysis (5). It is common for patients in this clinical condition to present endotoxemia and this, in parallel, further exacerbates renal dysfunction (6).

In addition, the release of inflammatory mediators during hemodialysis treatment is indicated as an aggravating factor for the increase in morbidity, mortality, and decline in the subjective perception of overall quality of life (7). In our study, we chose inflammatory markers that had already been described in other studies. Saraheimo et al. (8) demonstrated a close relationship between increased IL- 6 levels and C-reactive protein parallel to the severity of renal disease. The augment of this cytokine is gradual and according to the progress of CKD (9). Homocysteine (Hcy) is a thrombogenic factor that antagonizes nitric oxide. Besides, hyperhomocysteinemia represents a risk factor for cardiovascular diseases and mortality in patients with CKD, especially when associated with high levels of CRP (10).

Correspondence: M.R. Bacci: <mrbacci@yahoo.com> 
This information raised the need to search for possible correlations between the biochemical and inflammatory changes present in terminal CKD and some parameters of the KDQOL-SF36 questionnaire. The aim of the present study was to apply the KDQOL-SF36 adapted to the Portuguese language and to observe its association with inflammatory and biochemical markers.

\section{Material and Methods}

\section{Patients}

This was an analytical and descriptive observational study. All patients on hemodialysis treatment in Hospital Mário Covas and Centro de Hemodiálise de Mauá (SP, Brazil) were invited to take part in this study.

Patient inclusion criteria were: a) age greater than 21 years; b) confirmed diagnosis of end-stage CKD; and c) having had 3 hemodialysis sessions per week for at least 6 months.

The exclusion criteria were: a) systemic infection or hospitalization at the time of the interview; b) recent diagnosis or undergoing treatment for neoplasia; and c) psychiatric illness under treatment at the time of the interview. No patients were excluded from the study; all the interviewees met the inclusion criteria and thirty patients, after giving their written informed consent, answered the KDQOL-SF36 validated for the Portuguese language during the dialysis sessions. The survey was registered with the local Ethics Committee (No. 19795613.6.0000.0082/2013).

\section{Variables}

Blood samples were collected only once at the start of the hemodialysis session on the day of the interview. The inflammatory markers used were Hcy, ultra-sensitive C-reactive protein (CRP), interleukin 6 (IL-6), TNF- $\alpha$, and serum ferritin. The adequacy assessment of the hemodialysis was performed through the single pool $\mathrm{kt} / \mathrm{v}$ value of each session on the day of the interview. A value above 1.2 was considered a reference for adequacy.

For the estimation of glomerular filtration rate (GFR), the simplified Modification of Diet in Renal Disease formula was used and the result reported in $\mathrm{mL} \cdot \mathrm{min}^{-1} \cdot\left(1.73 \mathrm{~m}^{2}\right)^{-1}$.

The anthropometric measurements of each patient were performed by a single nutritionist using triceps skinfold, abdominal circumference, arm muscle circumference, and body mass index (BMI) $\left(\mathrm{kg} / \mathrm{m}^{2}\right)$. The weight used was the ideal dry weight in kilograms. The nutritional status of each patient was also assessed by the serum albumin value.

\section{Statistical analysis}

All variables were analyzed descriptively. For quantitative variables, analysis was performed by observing the minimum and maximum values, and the calculation of means, SD, and medians. For qualitative variables, absolute and relative frequencies were calculated.

Using the Shapiro-Wilk test, it was verified that the majority of the data did not present a normal distribution
$(P<0.05)$ and were therefore reported as medians and interquartile ranges. To analyze the association between the variables, the Spearman correlation test was used. Statistical significance was set at $P \leqslant 0.05$. The statistical package used was Stata version 12.0 (StataCorp LP, USA).

\section{Results}

The percentage and average values of the demographic, anthropometric, and nutritional characteristics of the patients are reported in Table 1.

The KDQOL-SF36 data of the items of interest are reported in Table 2 as medians (25-75th percentiles).

Among the inflammatory markers studied, the highest value was for ferritin, with a median of $305.5 \mathrm{ng} / \mathrm{mL}(224-$ 380). The medians for the other markers were TNF- $\alpha=$ $16.7 \mathrm{pg} / \mathrm{mL}$ (12.9-28.30), Hcy = $13.5 \mu \mathrm{mol} / \mathrm{L}$ (10.7-18.1), IL-6 = $12.5 \mathrm{pg} / \mathrm{mL}(5-18)$, and CRP = $12.5 \mathrm{mg} / \mathrm{L}(6-20)$.

Evaluation of anthropometric parameters was made through the measurement of abdominal circumference, body mass index (BMI), triceps skinfold, and arm circumference. For these parameters, the following medians were obtained: abdominal circumference of $90.5 \mathrm{~cm}$ (75-96); BMl of $25.2 \mathrm{~kg} /$ height $(\mathrm{m})^{2}$ (21.5-27.3); triceps

Table 1. Demographic, anthropometric, and nutritional characteristics of the study population.

\begin{tabular}{lc}
\hline Variable & Value \\
\hline Gender (M/F; \%) & $45 / 55$ \\
Age (years; average) & 41.03 \\
Ethnic group (Blacks/Caucasians; \%) & $43.3 / 56.4$ \\
Time in hemodialysis (months; average) & 52.5 \\
Kt/V (average) & 1.34 \\
Arterial hypertension (\%) & 93.3 \\
Diabetes (\%) & 23.3 \\
Etiology of chronic kidney disease (\%) & \\
Arterial hypertension & 43.5 \\
Diabetes & 13.3 \\
Glomerulonephritis & 10.0 \\
Autoimmune & 3.3 \\
Undefined & 26.6 \\
Chronic pyelonephritis & 3.3 \\
Interdialytic gain (\%) & \\
Up to 3 kg & 53.4 \\
From 3 to 5 kg & 36.6 \\
Above 5 kg & 10.0 \\
Nutritional status (\%) & \\
Malnourished & 3.4 \\
Eutrophic & 46.6 \\
Overweight & 40.0 \\
Obese & 10.0 \\
\hline
\end{tabular}

Data are reported as percentages or averages. 
Table 2. Medians for items of the kidney disease quality of life short form 36 (KDQOL-SF 36) questionnaire.

\begin{tabular}{lc}
\hline Quality of life & Median \\
& $(25-75 \%)$ \\
\hline Chronic renal failure - specific domains & \\
Patient satisfaction & $5.5(5-6)$ \\
Dialysis staff encouragement & $4(2-6)$ \\
Social support & $7(6-8)$ \\
Sleep & $16(13-18)$ \\
Sexual function & $2(2-3)$ \\
Quality of social interactions & $9(7-10)$ \\
Cognitive function & $5(3-7)$ \\
Work status & $3(3-3)$ \\
Burden of kidney disease & $14(11-16)$ \\
Effects of kidney disease & $18(13-20)$ \\
List of symptoms/problems & $16.5(14-25)$ \\
Total & 100 \\
KDQOL-SF-36 & \\
Mental health & $19(17-21)$ \\
Limitations due to emotional health problems & $5.5(4-6)$ \\
Social functioning & $6(6-6)$ \\
Energy/Fatigue & $15(17-13)$ \\
Pain & $3(2-4)$ \\
Perception of general health & $17(19-13)$ \\
Physical functioning & $27(24-28)$ \\
Limitations due to physical health problems & $7(6-8)$ \\
Total & 99.5 \\
\hline
\end{tabular}

skinfold thickness of $20 \mathrm{~cm}$ (15-25); and arm circumference of $22.3 \mathrm{~cm}(21.2-24.4)$.

Statistical correlations between Hcy levels and work status and between IL- 6 levels and limitations due to health problems were found. There was no relationship between the other indices evaluated (Table 3).

Associations were found between triceps skinfold thickness and work status $(\mathrm{P}=0.01)$, pain and triceps skinfold $(P=0.05)$, and between arm circumference and patient satisfaction $(P=0.03)$ and physical functioning $(P=0.04)$ (Table 4).

The association between inflammatory markers and dialysis adequacy parameters is described in Table 5. Hcy was correlated with serum creatinine $(P=0.002)$, GFR $(P=0.003)$, and dialysis time $(P=0.02)$.

Among the inflammatory markers and nutritional parameters, there was a correlation between TNF- $\alpha$ and albumin $(P=0.008)$, nutritional status $(P=0.04)$, and arm circumference adequacy $(P=0.01)$. TNF- $\alpha$ was the inflammatory marker most commonly associated with nutritional parameters. There was an association between ferritin and nutritional status $(P=0.03)$, and between IL-6 and arm circumference adequacy ( $P=0.02$; Table 6$)$.

There was no association between nutritional adequacy parameters and albumin with triceps skinfold thickness adequacy $(P=0.66)$, with arm circumference adequacy $(P=0.92)$, and with $B M I(P=0.37)$.

\section{Discussion}

CKD is commonly accompanied by a negative impact on patient perception of health. These factors may also reflect in other aspects such as nutrition, cognition, and psychosocial factors (11). The KDQOL-SF36 is an instrument for evaluating the perception of quality of life in patients with end-stage renal disease and has a score ranging from 0 to 100 , allowing subjective measurement of important variables for proper treatment follow-up (12).

Patients on hemodialysis commonly present weight gain during the interdialytic period (13). Our data demonstrated that $53.4 \%$ of the patients studied had an interdialytic weight gain of $3 \mathrm{~kg} ; 36.6 \%$ gained from 3 to $5 \mathrm{~kg}$ and $10 \%$ had a weight gain of over $5 \mathrm{~kg}$. In our evaluation, we found no correlation between weight gain and the other quality of life parameters as well as with inflammatory markers.

When evaluating the nutritional profile and weight gain of the individuals studied, we found that $3.4 \%$ of the patients were malnourished and $46.6 \%$ were classified as eutrophic. On the other hand, $40 \%$ were overweight and $10 \%$ were obese. Malnutrition apparently was not a predictive factor for worse quality of life in this population $(14,15)$.

Ferraz et al. (16), studying the nutritional status in patients submitted to hemodialysis, found a correlation between malnutrition and interdialytic weight gain. However, they emphasized that findings should not be generalized due to interferences of individual factors that may reflect on nutrition and weight.

Tannor et al. (17), in an evaluation of the quality of life in dialysis patients in South Africa, found that patients on dialysis have a poor quality of life regardless of the treatment being peritoneal dialysis or hemodialysis. The parameters that differentiated the analysis were scores of specific domains for each type of treatment, but in general, both therapies resulted in reduction of quality of life.

Almutary et al. (18) demonstrated that sexual dysfunction affects approximately one third of dialytic renal patients and is recurrent mainly in older individuals. On the other hand, it has been reported that dialysis patients have difficulty in feeling comfortable and having a satisfactory sex life due to the use of catheters and the discomfort caused by generalized pain (17). Thus, we believe that the pain variable is one of the preponderant factors that interferes in the other variables of quality of life perception.

Raghavan et al. (19) state that pain is a symptom that increases in parallel with the progression of kidney disease and grows exponentially as the patient ages. The authors suggest that these patients initiate pain-supportive care that does not affect renal disease progression. Moreover, pain may also reflect a limitation to perform essential 
Table 3. Spearman's correlation coefficients between quality of life and inflammatory markers.

\begin{tabular}{|c|c|c|c|c|c|}
\hline \multirow[t]{2}{*}{ Quality of life } & \multicolumn{5}{|c|}{ Inflammatory markers } \\
\hline & TNF- $\alpha$ & IL-6 & CRP & Hcy & Ferritin \\
\hline \multicolumn{6}{|l|}{ Chronic renal failure - specific domains } \\
\hline Patient satisfaction & 0.165 & 0.083 & -0.156 & -0.052 & 0.026 \\
\hline Dialysis staff encouragement & -0.146 & -0.034 & 0.358 & 0.053 & 0.214 \\
\hline Social support & 0.014 & 0.108 & 0.092 & -0.016 & -0.105 \\
\hline Sleep & 0.234 & -0.132 & -0.116 & -0.315 & 0.190 \\
\hline Sexual function & -0.182 & 0.038 & -0.132 & 0.031 & -0.031 \\
\hline Quality of social interactions & -0.036 & -0.110 & -0.038 & 0.053 & 0.100 \\
\hline Cognitive function & 0.075 & -0.004 & 0.084 & -0.279 & 0.033 \\
\hline Work status & 0.114 & 0.309 & 0.259 & $-0.374^{*}$ & -0.106 \\
\hline Burden of kidney disease & 0.171 & 0.075 & -0.019 & -0.346 & 0.055 \\
\hline Effects of kidney disease & 0.123 & 0.161 & 0.300 & 0.061 & 0.328 \\
\hline List of symptoms/problems & 0.032 & 0.064 & 0.202 & -0.026 & 0.144 \\
\hline \multicolumn{6}{|l|}{ KDQOL-SF 36} \\
\hline Mental health & -0.130 & -0.319 & 0.008 & 0.264 & 0.110 \\
\hline Limitations due to emotional health problems & -0.026 & -0.180 & -0.172 & -0.277 & -0.001 \\
\hline Social functioning & 0.023 & 0.268 & 0.313 & -0.136 & -0.007 \\
\hline Energy/Fatigue & 0.210 & -0.002 & 0.214 & 0.228 & -0.007 \\
\hline Pain & -0.035 & 0.084 & 0.184 & 0.042 & 0.061 \\
\hline Perception of general health & -0.161 & 0.108 & 0.205 & 0.021 & 0.022 \\
\hline Physical Functioning & -0.174 & -0.216 & -0.058 & 0.018 & -0.005 \\
\hline Limitations due to physical health problems & 0.358 & $0.404^{* *}$ & -0.147 & -0.266 & 0.023 \\
\hline
\end{tabular}

Data are reported as Spearman's correlation coefficient (rho). TNF- $\alpha$ : tumor necrosis factor alpha; IL-6: interleukin 6; CRP: ultrasensitive C-reactive protein; Hcy: homocysteine; KDQOL-SF 36: kidney disease quality of life short form 36 questionnaire. ${ }^{*} \mathrm{P}<0.05$; ${ }^{* *} \mathrm{P}<0.01$.

activities, as verified in the parameter "limitations due to physical health problems" (6-11).

The parameters with the best indices were: the "effects of kidney disease", "list of symptoms/problems", "sleep", and the "perception of the burden of kidney disease". The variables "cognitive function", "limitations due to emotional health problems", and "social functioning" obtained the lowest scores. Studies have suggested that one of the possible determinants of cognition loss in these patients is the decline in GFR and increased synthesis of proinflammatory mediators $(1,20-22)$.

A study in a rat model of chronic renal failure by $5 / 6$ nephrectomy found an elevation of proinflammatory cytokines. The authors suggest that this increase is related to the decrease in cognition in CKD due to the detection of proinflammatory cytokines in specific nuclei of the central nervous system (23).

Our study found that Hcy was correlated with the "work status" variable and IL-6 levels with the variable "limitations due to health problems". Hcy participates in the physiopathology of cardiovascular diseases (23), Alzheimer's (24), depression (25), and in kidney disease (26). All of these pathologies are directly involved in loss of quality of life and limitations due to these health problems, which may directly reflect on performance at work and other social activities.

When analyzing the relationship between anthropometric data and the same inflammatory mediators, we found that there was a significant correlation between abdominal circumference and ferritin, and between BMI and both CRP and ferritin values. "Triceps skinfold thickness" was correlated with values of TNF- $\alpha$. There is no data in the literature on the relationship between measurements of abdominal circumference or BMI and ferritin levels in end-stage renal patients. It is possible that the nutritional status of the studied population directly influenced this interaction.

There is evidence indicating the participation of hyperhomocysteinemia in the pathophysiology of kidney disease (26). Foucher et al. (27), reporting the relationship between Hcy and serum creatinine levels, showed that the increase in Hcy is much greater than the elevation of creatinine, making it clear that Hcy alterations progress more rapidly than alterations in serum creatinine levels in kidney patients. The authors also report that these factors may contribute significantly to an increased mortality rate and they attribute this to the increased release of inflammatory mediators. 
Table 4. Spearman's correlation coefficients between quality of life and anthropometric parameters.

\begin{tabular}{|c|c|c|c|c|}
\hline \multirow[t]{2}{*}{ Quality of life } & \multicolumn{4}{|c|}{ Anthropometric parameters } \\
\hline & Abdominal circumference & BMI & Triceps skinfold & Arm circumference \\
\hline \multicolumn{5}{|l|}{ Chronic renal failure - specific domains } \\
\hline Patient satisfaction & -0.254 & -0.136 & 0.124 & $-0.378^{*}$ \\
\hline Dialysis staff encouragement & 0.302 & 0.114 & -0.311 & 0.318 \\
\hline Social support & 0.033 & -0.161 & 0.253 & -0.322 \\
\hline Sleep & -0.231 & -0.150 & -0.204 & -0.053 \\
\hline Sexual function & -0.193 & -0.298 & -0.325 & -0.085 \\
\hline Quality of social interactions & -0.193 & -0.011 & 0.141 & -0.207 \\
\hline Cognitive function & 0.011 & -0.013 & -0.031 & -0.123 \\
\hline Work status & 0.072 & 0.230 & $0.467^{\star \star *}$ & -0.089 \\
\hline Burden of kidney disease & 0.025 & 0.059 & 0.116 & 0.099 \\
\hline Effects of kidney disease & 0.016 & 0.166 & -0.090 & -0.072 \\
\hline List of symptoms/problems & -0.233 & 0.036 & -0.136 & 0.029 \\
\hline \multicolumn{5}{|l|}{ KDQOL-SF 36} \\
\hline Mental health & 0.161 & 0.269 & 0.149 & 0.121 \\
\hline Limitations due to emotional health problems & -0.231 & -0.146 & -0.019 & -0.018 \\
\hline Social functioning & 0.096 & 0.051 & 0.165 & 0.266 \\
\hline Energy/Fatigue & 0.229 & 0.354 & 0.282 & 0.044 \\
\hline Pain & 0.075 & 0.011 & $-0.493^{\star *}$ & 0.335 \\
\hline Perception of general health & 0.103 & 0.022 & 0.044 & 0.233 \\
\hline Physical Functioning & -0.158 & -0.254 & 0.084 & $-0.370^{*}$ \\
\hline Limitations due to physical health problems & -0.120 & -0.006 & 0.197 & -0.047 \\
\hline
\end{tabular}

Data are reported as Spearman's correlation coefficient (rho). BMI: body mass index; KDQOL-SF 36: kidney disease quality of life short form 36 questionnaire. ${ }^{*} \mathrm{P}<0.05 ;{ }^{* *} \mathrm{P}<0.01$; ${ }^{* *} \mathrm{P}<0.001$.

Table 5. Spearman's correlation coefficients between inflammatory markers and dialysis adequacy parameters.

\begin{tabular}{lccccccc}
\hline Inflammatory markers & \multicolumn{7}{c}{ Dialysis adequacy parameters } \\
\cline { 2 - 8 } & Kt/v & Urea & Creatinine & GFR & Number of fistulas & Number of catheters & Dialysis time \\
\hline TNF- $\alpha$ & 0.203 & -0.155 & -0.155 & 0.113 & 0.031 & 0.136 & -0.079 \\
IL-6 & -0.132 & -0.067 & -0.180 & 0.123 & -0.295 & -0.024 & -0.118 \\
CRP & -0.163 & 0.015 & 0.150 & -0.176 & -0.199 & 0.09 & $\mathbf{- 0 . 4 0 1}^{*}$ \\
Hcy & -0.175 & 0.257 & $\mathbf{0 . 5 3 8 ^ { * * }}$ & $-\mathbf{0 . 5 1 8 ^ { * * }}$ & 0.173 & 0.149 & $\mathbf{0 . 4 1 1}^{*}$ \\
Ferritin & -0.115 & 0.047 & 0.126 & -0.170 & -0.051 & 0.217 & 0.145 \\
\hline
\end{tabular}

Data are reported as Spearman's correlation coefficient (rho). GFR: glomerular filtration rate; TNF- $\alpha$ : tumor necrosis factor alpha; IL-6: interleukin 6; CRP: ultra-sensitive C-reactive protein; Hcy: homocysteine. ${ }^{*} \mathrm{P}<0.05 ;{ }^{* \star} \mathrm{P}<0.01$.

Table 6. Spearman's correlation coefficients between inflammatory markers and nutritional parameters.

\begin{tabular}{lcccc}
\hline Inflammatory markers & \multicolumn{3}{c}{ Nutritional parameters } \\
\cline { 2 - 5 } & Albumin & Nutritional status & Triceps skinfold adequacy & Arm circumference adequacy \\
\hline TNF- $\alpha$ & $-\mathbf{0 . 4 7 4}^{*}$ & $\mathbf{0 . 3 6 1 *}^{*}$ & $\mathbf{0 . 3 7 1 ^ { * }}$ & -0.128 \\
IL-6 & $-\mathbf{0 . 4 2 4}^{*}$ & 0.224 & 0.353 & -0.002 \\
CRP & $-\mathbf{0 . 3 7 5 ^ { * }}$ & $0.419^{*}$ & $\mathbf{0 . 4 9 0 ^ { * * }}$ & 0.308 \\
Hcy & 0.079 & 0.178 & -0.194 & 0.200 \\
Ferritin & -0.162 & $\mathbf{0 . 3 8 8 *}^{*}$ & -0.131 & 0.153 \\
\hline
\end{tabular}

Data are reported as Spearman's correlation coefficient (rho). TNF- $\alpha$ : tumor necrosis factor alpha; IL-6: interleukin 6; CRP: ultrasensitive C-reactive protein; Hcy: homocysteine. ${ }^{*} \mathrm{P}<0.05$; ${ }^{* \star} \mathrm{P}<0.01$. 
Although the observed results contribute to future assessments of quality of life in patients during hemodialysis treatment, it should be noted that the number of patients studied may not allow generalization of the findings. In addition, a one-time blood sample from patients treated in a single center may limit the generalizability and reliability of the results. Multiple hypotheses were tested,

\section{References}

1. Elias MF, Elias PK, Seliger SL, Narsipur SS, Dore GA, Robbins MA. Chronic kidney disease, creatinine and cognitive functioning. Nephrol Dial Transplant 2009; 24: 24462452, doi: 10.1093/ndt/gfp107.

2. Braga SF, Peixoto SV, Gomes IC, Acurcio Fde A, Andrade El, Cherchiglia ML. Factors associated with health-related quality of life in elderly patients on hemodialysis. Rev Saude Publica 2011; 45: 1127-1136, doi: 10.1590/S0034-89102 011000600015.

3. Ware JE Jr, Sherbourne CD. The MOS 36-item short-form health survey (SF-36). I. Conceptual framework and item selection. Med Care 1992; 30: 473-483, doi: 10.1097/0000 5650-199206000-00002.

4. Sousa-Martins P, Moura A, Madureira J, Alija P, Oliveira JG, Lopez $M$, et al. Risk factors for mortality in end-stage kidney disease patients under online-hemodiafiltration: three-year follow-up study. Biomarkers 2016; 21: 544-550, doi: 10.3109/ 1354750X.2016.1160428.

5. Kalantar-Zadeh K, Block G, McAllister CJ, Humphreys MH, Kopple JD. Appetite and inflammation, nutrition, anemia, and clinical outcome in hemodialysis patients. Am J Clin Nutr 2004; 80: 299-307, doi: 10.1093/ajcn/80.2.299.

6. Hassan MO, Duarte R, Dix-Peek T, Vachiat A, Naidoo S, Dickens $\mathrm{C}$, et al. Correlation between volume overload, chronic inflammation, and left ventricular dysfunction in chronic kidney disease patients. Clin Nephrol Supplement 2016; 86: 131-135, doi: 10.5414/CNP86S127.

7. Lai S, Mecarelli O, Pulitano P, Romanello R, Davi L, Zarabla $A$, et al. Neurological, psychological, and cognitive disorders in patients with chronic kidney disease on conservative and replacement therapy. Medicine (Baltimore) 2016; 95: e5191, doi: 10.1097/MD.0000000000005191.

8. Saraheimo M, Teppo AM, Forsblom C, Fagerudd J, Groop $\mathrm{PH}$. Diabetic nephropathy is associated with low-grade inflammation in Type 1 diabetic patients. Diabetologia. 2003; 46: 1402-1407, doi: 10.1007/s00125-003-1194-5.

9. Tong M, Carrero JJ, Qureshi AR, Anderstam B, Heimbürger $\mathrm{O}$, Bárány $\mathrm{P}$, et al. Plasma pentraxin 3 in patients with chronic kidney disease: associations with renal function, protein-energy wasting, cardiovascular disease, and mortality. Clin J Am Soc Nephrol 2007; 2: 889-897, doi: 10.2215/ CJN.00870207.

10. Pastore A, De Angelis S, Casciani S, Ruggia R, Di Giovamberardino G, Noce A, et al. Effects of folic acid before and after vitamin B12 on plasma homocysteine concentrations in hemodialysis patients with known MTHFR genotypes. Clin Chem 2006; 52: 145-148, doi: 10.1373/ clinchem.2005.056119.

11. Duarte PS, Miyazaki MC, Ciconelli RM, Sesso R. Translation and cultural adaptation of the quality of life assessment and the small sample size did not allow correcting P-values. Therefore, our study may have a high probability for global type I error.

In conclusion, Hcy was directly related to nutritional status, creatinine, and inflammatory markers. In the studied population, most sections of the KDQOL-SF36 were not correlated with inflammatory cytokine levels.

instrument for chronic renal patients (KDQOL-SF). Rev Assoc Med Bras 1992; 49: 375-381, doi: 10.1590/S0104-423020 03000400027.

12. Glover C, Banks P, Carson A, Martin CR, Duffy T. Understanding and assessing the impact of end-stage renal disease on quality of life: a systematic review of the content validity of self-administered instruments used to assess health-related quality of life in end-stage renal disease. Patient 2011; 4: 19-30, doi: 10.2165/11584650-00000000000000.

13. Sakai MA, Hamada H, Hara K, Mori K, Uchida MT, Mizuguchi Md T, et al. Nutritional counseling regulates interdialytic weight gain and blood pressure in outpatients receiving maintenance hemodialysis. J Med Invest 2017; 64: 129-135, doi: 10.2152/jmi.64.129.

14. Ifudu O, Uribarri J, Rajwani I, Vlacich V, Reydel K, Delosreyes $\mathrm{G}$, et al. Relation between interdialytic weight gain, body weight and nutrition in hemodialysis patients. Am J Nephrol 2002; 22: 363-368, doi: 10.1159/0000 65228.

15. Bots CP, Brand HS, Veerman EC, Valentijn-Benz M, Van Amerongen BM, Valentijn RM, et al. Interdialytic weight gain in patients on hemodialysis is associated with dry mouth and thirst. Kidney Int 2004; 66: 1662-1668, doi: 10.1111/j.15231755.2004.00933.x

16. Ferraz SF, Freitas AT, Vaz IM, Campos MI, Peixoto Mdo R, Pereira ER. Nutritional status and interdialytic weight gain of chronic hemodialysis patients. J Bras Nefrol 2015; 37: 306-314, doi: 10.5935/0101-2800.20150050.

17. Tannor EK, Archer E, Kapembwa K, van Schalkwyk SC, Davids MR. Quality of life in patients on chronic dialysis in South Africa: a comparative mixed methods study. BMC Nephrol 2017; 18: 4, doi: 10.1186/s12882-0160425-1.

18. Almutary $\mathrm{H}$, Bonner $\mathrm{A}$, Douglas $\mathrm{C}$. Which Patients with chronic kidney disease have the greatest symptom burden? A comparative study of advanced ckd stage and dialysis modality. J Ren Care 2016; 42: 73-82, doi: 10.1111/jorc.12152.

19. Raghavan D, Holley JL. Conservative Care of the Elderly CKD Patient: A practical guide. Adv Chronic Kidney Dis 2016;23: 51-56, doi: 10.1053/j.ackd.2015.08.003.

20. Davey A, Elias MF, Robbins MA, Seliger SL, Dore GA. Decline in renal functioning is associated with longitudinal decline in global cognitive functioning, abstract reasoning and verbal memory. Nephrol Dial Transplant 2013; 28: 1810-1819, doi: 10.1093/ndt/gfs470.

21. Menkes DL, Buchman AS, Shah RJ, Boyle PA, Bennett DA. Kidney function is associated with the rate of cognitive decline in the elderly: Neurology 2010; 74: 1656, doi: 10.1212/WNL. Ob013e3181d8a4ef. 
22. Degaspari S, Tzanno-Martins CB, Fujihara CK, Zatz R, Branco-Martins JP, Viel TA, et al. Altered KLOTHO and NF-kappaB-TNF-alpha signaling are correlated with NephrectomyInduced cognitive impairment in rats. PLoS One 2015; 10: 2015, doi: 10.1371/journal.pone.0125271.

23. Baggott JE, Tamura T. Homocysteine, iron and cardiovascular disease: a hypothesis. Nutrients 2015; 7: 1108-1118, doi: 10.3390/nu7021108.

24. Kamat PK, Vacek JC, Kalani A, Tyagi N. Homocysteine induced cerebrovascular dysfunction: a link to Alzheimer's disease etiology. Open Neurol J 2015; 9: 9-14, doi: 10.2174/ $1874205 \times 01509010009$.
25. de Koning EJ, van der Zwaluw NL, van Wijngaarden JP, Sohl E, Brouwer-Brolsma EM, van Marwijk HW, et al. Effects of two-year Vitamin B12 and Folic Acid supplementation on depressive symptoms and quality of life in older adults with elevated homocysteine concentrations: additional results from the B-PROOF Study, an RCT. Nutrients 2016; 8: pii: E748, doi: $10.3390 /$ nu8110748.

26. Long Y, Nie J. Homocysteine in Renal Injury. Kidney Dis (Basel) 2016; 2: 80-87, doi: 10.1159/000444900.

27. Foucher $C$, Brugère $L$, Ansquer JC. Fenofibrate, homocysteine and renal function. Curr Vasc Pharmacol 2010; 8: 589-603. Review, doi: 10.2174/157016110792006987. 